\title{
Clinical Acid-Base Pathophysiology: Disorders of Plasma Anion Gap
}

\author{
Orson W. Moe $e^{1,2,3}$ and Daniel Fuster ${ }^{1}$ \\ Department of Internal Medicine ${ }^{1}$ and \\ Center of Mineral Metabolism and Clinical Research ${ }^{2}$, \\ University of Texas Southwestern Medical Center \\ Medical Service, Department of Veterans Affairs Medical Center ${ }^{3}$ \\ Dallas, Texas, U.S.A.
}

Key words: Acid-base. Anion Gap. Pyroglutamic acid

Address correspondence to:

Orson W. Moe, M.D.

Department of Internal Medicine

University of Texas Southwestern Medical Center

5323 Harry Hines Blvd

Dallas, TX 75390-8856

tel 214-648-3152

fax 214-648-2071

email: orson.moe@utsouthwestern.edu 


\begin{abstract}
The plasma anion gap is a frequently used parameter in the clinical diagnosis of a variety of conditions. The commonest application of the anion gap is to classify metabolic acidosis into those that do or do not leave unmeasured anions in the plasma. While this algorithm is useful in streamlining the diagnostic process, it should not be used solely in this fashion. The anion gap measures the difference between the unmeasured anions and unmeasured cations and thus conveys much more information to the clinician than just quantifying anions of strong acids. In this review, the significance of the anion gap is emphasized and several examples are given to illustrate a more analytic approach to using the clinical anion gap. These include disorders of low anion gap, respiratory alkalosis and pyroglutamic acidosis.
\end{abstract}




\section{Practice Points}

- The anion gap measures the difference between all unmeasured anions other than $\mathrm{HCO}_{3}{ }^{-}$ and $\mathrm{Cl}^{-}$, and all unmeasured cations other than $\mathrm{Na}^{+}$.

- The clinical utility of the anion gap extends far beyond the differential diagnosis of metabolic acidosis.

- It is advisable that the clinician knows the normal range of the anion gap and the assays used in measuring $\mathrm{Na}^{+}$and $\mathrm{Cl}^{-}$in the laboratories supporting their practice.

- The ability to suspect and recognize that the anion gap is higher or lower than expected in a given clinical scenario allows the clinician to have much wider, sharper and more sophisticated diagnostic capabilities with information that is presented in routine chemistry reports. 


\section{Research Agenda}

- $\quad$ The role of strong (fully dissociated at plasma $\mathrm{pH}$ ) ions and weak (titratable with $\mathrm{H}^{+}$at plasma $\mathrm{pH}$ ) cations and anions in determining in acid-base physiology is often unrecognized. This area deserves further data collection in whole organism physiologic and pathophysiologic settings.

- There are vast unexplored territories in how physiologic and pathophysiologic conditions alter unmeasured cation and anion concentrations in terms of their generation, metabolism, and renal handling. Work in these areas is likely to be fruitful in generating novel knowledge in acid-base-electrolyte physiology and clinical medicine.

- Pyroglutamic acidosis, particularly in milder forms, are more common than realized. The pathophysiology of the acquired form of this condition is not clear. Animal models as well as carefully performed metabolic studies in humans are much needed to understand this potentially life-threatening condition. It is likely that there are other forms of acidbase disorders that are unrecognized 


\section{Introduction}

The plasma anion gap is a calculated entity that is utilized frequently in clinical practice and is automatically displayed in most routine chemistry reports. The anion gap assumes the most central decisive role in the diagnostic algorithms of metabolic acidosis pontificated by most textbooks. An implicit axiom is that diagnosticians have secured sufficient expertise in interpreting the biologic significance of disturbances in plasma anion gaps and are apt in translating this knowledge into clinical actions. The commonest wisdom derived from an elevated anion gap is that the addition of acid $\left(\mathrm{H}^{+} \mathrm{A}^{-}\right)$into the blood has left a footprint because the anion $\left(\mathrm{A}^{-}\right)$has remained unaltered in the plasma. While this foremost conclusion is by-andlarge valid, proficiency in the interpretation of anion should extent beyond the ability to arrive at this resolution. The scope of the topic precludes an exhaustive discussion of all aspects of the plasma anion gap in a single monograph. Selected aspects of the plasma anion gap are reviewed to improve the understanding of this simple clinical chemical data. After a brief treatise on the physiology of the anion gap, this review will focus on three clinical scenarios; low anion gap, anion in respiratory alkalosis, and the increasingly recognized condition of pyroglutamic acidosis.

\section{Determinants and Significance of the Plasma Anion Gap}

Although inaccurate, the term anion gap is commonly accepted in everyday clinical parlance. As charge balance precludes the existence of any gaps, the more accurate term should really be "difference between unmeasured anions and unmeasured cations" which obviously lacks the brevity requisite for practical communication. The arbitrary relationship between the concentrations of three commonly measured ions $\left(\mathrm{Na}^{+}-\mathrm{Cl}^{-}-\mathrm{HCO}_{3}{ }^{-}\right)$constitutes the clinical "anion gap". It is important to point out that this entity actually equals [anionic proteins + 
inorganic phosphate + sulfate + organic anions] minus [potassium + calcium + magnesium + cationic proteins]; all in milliequivalents. Since there are more unmeasured anions than unmeasured cations, this term is usually positive. The analysis of the various components has been covered in previous reviews $(1,2)$.

The classical numeric value of a normal anion gap is considered $12 \pm 4 \mathrm{mEq} / \mathrm{L}$ based on studies conducted in the 1970's, when sodium was determined by flame photometry (based on the principle of flame atomic emission spectrometry) and chloride by a colorimetric assay (mercuric-nitrate-thyocyanate colorimetric assay). In the early 1980's, ion selective electrodes for specific ionic species were introduced for the measurement of serum electrolytes. The difference between the ionic concentration in the electrode (known) and the sample creates an electrical potential (measured) and the sample ionic concentration can be calculated. This new method has caused a shift of the AG from $12 \pm 4 \mathrm{mEq} / \mathrm{L}$ down to $6 \pm 3 \mathrm{mEq} / \mathrm{L}$. (3). It is a worthwhile effort for clinicians to be acquainted with the range of normal anion gap and the measuring methods of serum sodium and chloride in the laboratories that support their practice. Since there are significant inter-individual variations of anion gap, comparing the temporal intra-individual changes in anion gap may sometimes be more useful.

A point made earlier is that an elevated anion gap does not merely reflect the anion $\mathrm{A}^{-}$ remaining in the plasma after $\mathrm{H}^{+} \mathrm{A}^{-}$was added with decomposition of $\mathrm{HCO}_{3}^{-}$. The following analysis highlights this notion. Table 1 illustrates one of many lessons one can learn from nonmammalian physiology. Consider a quintessential anion gap metabolic acidosis- pure lactic acidosis- but with two differences. The lactic acidosis though massive, is physiologic and the subject is not a human but a diving turtle. Table 1 shows some plasma values in a painted turtle before a dive and 150 days later in an anoxic aquatic environment that is completely physiologic for this species (4). The resultant prolonged anaerobic metabolism generates a colossal amount of lactic acid. The usual analysis by a human biologist (a physician) would take the increase in 
anion gap $(\Delta=34$, Table 1$)$ which roughly approximates the fall in bicarbonate $(\Delta=45$, Table 1$)$ and assumes these changes represent the footprint of the lactate added which is equivalent to the amount of decomposed bicarbonate. A perusal of the actual measurements clearly demonstrates that this analysis is very far from the truth (Table 1). Lactate has actually gone up by $181 \mathrm{mM}$ instead of the $34-45 \mathrm{mM}$ predicted from the $\Delta$ anion gap. The decrement in plasma bicarbonate is much lower than the addition of lactate because most of the $\mathrm{H}^{+}$from lactic acid is buffered by the carbonate/bicarbonate in the shell rather than plasma buffer. In addition, the cations released from the shell into the plasma in exchange for the $\mathrm{H}^{+}$greatly elevated the unmeasured cations. This example illustrates: 1. When buffers outside the extracellular fluid are used, a strong acid will not result in equivalence in fall in bicarbonate and rise in the anion gap. 2. Simultaneous increase in unmeasured anions and cations has a nullifying effect on each other in terms of the value of the anion gap. This exercise serves as caution against the reflex conclusion that the rise in anion gap equals the added anion from the invading acid, which equals the fall in bicarbonate. For those who share the belief that physicians have much to learn from non-mammalian biology, several excellent monographs are available for further reading about physiologic lactic acidosis and shell buffering in turtles (4-6).

The clinical conception and application of the anion gap treats it as a passive parameter in the sense that it merely reflects the replacement of $\mathrm{HCO}_{3}^{-}$with an anion from a strong acid. Every textbook provides a list of strong acid suspects. Note that not all acid-base physiologists share this view. First touched upon by Lawrence Henderson, later fully espoused and presented by Paul Stewart in 1983 (7), and in modified forms subsequently (8), is the concept of strong ion difference. The strong ion difference hypothesis proposed three independent determinants of plasma $\mathrm{pH}$ : 1. $\mathrm{CO}_{2}$ tension, 2. Strong ion (fully dissociated ions at physiologic $\mathrm{pH}$ ) difference which is the sum of sodium, potassium, calcium, magnesium minus the sum of chloride, lactate, sulphate, ketoanions, and fatty acids. 3. Anions titratible with $\mathrm{H}^{+}$at physiologic $\mathrm{pH}$ such as phosphate, albumin, and globulins. Note that the terms listed under 2 and 3 are all reflected in the 
clinical anion gap equation. From Stewart's strong ion difference hypothesis, one garners a different panorama of the clinical anion gap as this entity assumes the role of an independent variable rather than just a sentinel. This review will not expound this alternative position but the interested reader can find such discussions by Constable, Boyle, and Baldwin $(9,10)$.

\section{Approach to a Low Anion Gap}

Whereas the presence of a high anion gap educes the consideration of a differential diagnosis by reflex in daily clinical practice, a low anion gap often does not elicit the same warning to clinicians and hence often remains either undiscovered or neglected. Furthermore, the classic differential diagnosis of a low anion gap has changed since ion selective electrode has been introduced. A low anion gap has two utilities. First, it can be an early and sometimes only sign of an underlying disease process such as paraproteinemia. Second, a low anion gap can mask an underlying high anion gap acidosis and potentially delay intervention. While an increase in the anion gap is almost always caused by retained unmeasured anions, a decrease in the anion gap can be generated by multiple mechanisms.

Errors in plasma sodium. Acute dilution of the extracellular fluid with either administered or retained hypotonic fluid will theoretically dilute essentially all ions causing a fall in the anion gap. However, this type of dilution usually results only in reductions of the anion gap of 1 or $2 \mathrm{mEql} / \mathrm{L}$ which will hardly move it out of the laboratory normal range. Severe hypernatremia can be underestimated by flame photometry due to the non-linear nature of this assay. Since the measurement of plasma chloride and bicarbonate remains accurate even at increased concentrations, the net result will be a spuriously low anion gap $(11,12)$. The use of sodium-selective electrodes with a larger dynamic range negates this artifact from instrument non-linearity. Large non-aqueous volumes in the plasma such as hypertriglyceridemia or dysproteinemia (12-16) can lead to an underestimation of serum sodium especially with flame 
photometric instruments. However, sodium-selective electrodes are not spared even though the sodium activity registered by the sodium electrode is oblivious to the non-aqueous volume. This is explained in Figure 1. Automatic aspirators and dilutors prepare the samples and in situations with excessive non-aqueous volume in plasma, a smaller than expected plasma volume may be aspirated leading to an underestimation of plasma sodium during the back-calculation (Fig 1). In the absence of concurrent errors in $\mathrm{Cl}^{-}$and $\mathrm{HCO}_{3}{ }^{-}$measurements, a low anion gap ensures. In macroglobulinemia, the error in sodium measurement is proportionate to the level of hyperviscosity and can be as high as $40 \mathrm{mEql} / \mathrm{L}$. (17)

Hypoalbuminemia. At a normal serum $\mathrm{pH}$ of 7.4, majority of the plasma proteins are anionic. Albumin with an average negative charge of 18 per mole at physiologic $\mathrm{pH}$ has been shown to be responsible for approximately $75 \%$ of the unmeasured anions of the normal anion gap (18). A drop in albumin by $10 \mathrm{~g} / \mathrm{L}$ therefore will cause the anion gap to fall by approximately $2.5 \mathrm{mEq} / \mathrm{L}$ at constant $\mathrm{pH}(19,20)$. Hypoalbuminemia is probably the commonest cause of a clinically relevant lowered anion gap. When both low anion gap and hypoalbuminemia are present, it is prudent to ensure that the low anion gap can be quantitatively accounted for by the low albumin.

Errors in plasma chloride. Hypertriglyceridemia can interfere with the colorimetric assays of serum chloride measurements due to light-scattering effects and result in marked overestimation of serum chloride. Overestimation of serum chloride by lipid interference does not occur with ion selective electrode measurements (14). Chloride is normally the only halide present in the extracellular fluid in significant concentrations. In acute or chronic bromide ingestion, bromide slowly replaces the extracellular chloride mainly because of their differential renal tubular handling; the renal tubule seems to reabsorb bromide more avidly than chloride, leading to its accumulation in the extracellular fluid (21). Classic sources of bromide were the bromide containing over-the-counter sedatives. More recently, pyridostigmin bromide used in 
the treatment of myasthenia gravis has been reported to cause bromide intoxication. (22). The effect of plasma bromide on the anion gap depends on the methods of measurement of chloride (Table 1). In the titrametrical measurement assay, both halides react identically and there will be no change in the anion gap with high plasma bromide concentration (23). With the colorimetric assays, not only are the two halides indistinguishable, bromide usually yields a deeper color than chloride giving rise an artificially high chloride concentration resulting in a low anion gap (24). With highly selective methods such as voltammetry (25), bromide and chloride does not crossreact and bromide intoxication presents with an elevated anion gap. By the same mechanism as bromide, another halide, iodide, can lead to changes anion gap depending on the assay methods (26). Iodide is found in radiologic contrast material expectorants and a number of other medications. Patients with renal insufficiency are prone to retain iodide and develop iodinism.

Non-sodium cations. In addition to displacement of sodium-containing water from serum by large amounts of non-sodium-containing paraproteins, some paraproteins can have a net positive charge at physiologic $\mathrm{pH}$. This leads to an increase of unmeasured cations and a low anion gap (1). Murray et al. found an correlation between the plasma anion gap and paraprotein levels and net positive charge on paraproteins in the patients with the lowest anion gaps (27). Of the two commonest immunoglobulins in myeloma, IgG tends to be cationic while IgA tends to be anionic at physiologic plasma $\mathrm{pH}$ and the disturbances in anion gap seems to correspond accordingly (IgG low gap; IgA high gap) (28-30) although this correlation is not always invariable (27-32). One convincing piece of evidence supportive of causality is the fact that chemotherapy results in simultaneous normalization in paraprotein and anion gap (33). Concomitant severe hypercalcemia and hypoalbuminemia are often contributing factors to a low anion gap in multiple myeloma.

Parenteral administration of the now rarely used cationic peptide antibiotic polymyxin B can result in hyperchloremia and thereby markedly reduce the serum anion gap. (34). Since the 
only cation included in the anion gap calculation is sodium, severe hyperkalemia, hypercalcemia, hypermagnesemia or lithium intoxication theoretically can also lead to a significantly decreased anion gap. Potassium and lithium are fully dissociated while calcium and magnesium exist in complexed forms that may or may not contribute cationic charges. For example, albumin bound calcium is uncharged but the calcium trivalent citrate soluble complex is a univalent anion. The key is to perform the correct calculation when assessing whether these elevated cations can quantitatively account for the low anion gap.

\section{The Anion Gap in Respiratory Alkalosis}

Not an uncommon consultation is one requesting evaluation of the following set of clinical chemistry data. In mM: $\mathrm{Na} 138, \mathrm{Cl} 103, \mathrm{HCO}_{3}{ }^{-} 18$, yielding an anion gap of 17 . Since the $\mathrm{HCO}_{3}$ has fallen about the same degree as the anion gap has risen, the clinician may algorithmically default to asking which acid consumed the $\mathrm{HCO}_{3}{ }^{-}$and left the footprint and start searching for an anion gap metabolic acidosis. This approach is clearly too restricted. The pitfall results from the low plasma bicarbonate from renal compensation of respiratory alkalosis, the unknown normal baseline anion gap of that individual, and the slightly elevated anion gap encountered in alkalemia.

Renal compensation in the form of $\mathrm{HCO}_{3}^{-}$excretion to reduce the plasma $\mathrm{HCO}_{3}^{-}$in response to a low systemic $\mathrm{pCO}_{2}$ is well documented involving both the proximal $(35,36)$ and distal $(37,38)$ nephrons. The mechanism by which this happens is unknown although one presumes a low $\mathrm{pCO}_{2}$ pervades throughout the body and causes an intracellular alkalosis in the renal epithelial cell. Since a fall in plasma $\mathrm{HCO}_{3}^{-}$can potentially rectify the plasma $\mathrm{pH}$ completely and the kidney is amply able to achieve that, it is unknown why the correction is always partial (i.e. plasma $\mathrm{pH}$ is never returned to normal). Since these issues are beyond the realm of this review, one will settle for the empirical existence of a low plasma $\mathrm{HCO}_{3}{ }^{-}$in our 
current discussion. We will focus on the plasma anion gap in alkalemia (high plasma $\mathrm{pH}$ ). Three mechanisms can contribute to raising the plasma anion gap in alkalemia. Each may be small in magnitude but in combination, these can explain the $3-4 \mathrm{mEq} / \mathrm{L}$ rise encountered in clinical situations.

A small but significant elevation of plasma anion gap has been repeatedly observed in chronic respiratory alkalosis in humans (39-42). The question remains as to what is the source of this alteration. One proposed mechanism is increased plasma lactate generation. This theory is based partly on the observation that acute hyperventilation raises plasma lactate levels (43-49). The rise in plasma lactate was quite variable in these experimental conditions and in some did not persist into the chronic phase. Nonetheless, these observations in conjunction with the biochemical evidence of stimulation of glycolysis by high $\mathrm{pH}$ have gathered popularity for this theory. It is prudent to point out two facts. First, even in the studies quoted above that clearly demonstrated elevated anion gap in respiratory alkalosis, it is not always possible to correlate the increased anion gap with elevated lactate levels. Second, the stimulation of phosphofructose kinase by high $\mathrm{pH}$ is well documented in vitro but how much does the glycolytic flux (hence lactate generation) actually go up in the intact cell and whole organism is not well established.

Part of the renal response to acute hypercapnia is bicarbonaturia which mandates mainly $\mathrm{Na}^{+}$and to a lesser extent $\mathrm{K}^{+}$to accompany the $\mathrm{HCO}_{3}$ in the urine. The loss of $\mathrm{NaHCO}_{3}$ leads to extracellular fluid volume contraction and raises plasma albumin concentration. However, if one calculates the magnitude of this increase in albumin concentration based on the typical renal bicarbonaturic response and acute volume of distribution of $\mathrm{HCO}_{3}^{-}$, the rise in anion gap contributed by this component will rarely exceed 1-2 $\mathrm{mEq} / \mathrm{L}$. In addition, hyperalbuminemia is an inconsistent finding in respiratory alkalosis (39-42).

The third mechanism by which alkalemia can raise plasma anion gap is via titration of 
plasma buffers. Albumin is frequently quoted as the main contributor of its relative abundance. The situation is far more complex in reality. First is the fact that albumin concentration itself per se can impact on plasma acid-base status as mentioned above via the Stewart mechanism (7, 9, 10). The group of Fencl presented experimental data of this effect and termed it "hypoproteinemia alkalosis" $(50,51)$. Regardless of this interaction, the question remains as to whether charge titration on albumin alone is sufficient to raise the anion gap. Non-denatured albumin has an isoelectric point of 5 and has a reasonably steep charge-to-pH relationship throughout $\mathrm{pH}$ 5-9 (52). However, the actual contribution of albumin to the change in anion gap observed with $\mathrm{pH}$ changes is quantitatively less than that predicted from titration of negative charges on albumin. Paulson made actual measurements and reviewed the literature and came out with an empiric relationship of $0.51 \mathrm{mEq} / \mathrm{L}$ of $\Delta$ anion gap for each $\Delta \mathrm{pH}$ of 0.1 units (53). This further emphasizes the fact that changes in $\mathrm{pH}$ alter charges on any anion or cation provided these ions do not exist perpetually in dissociation. These are ions in Stewart's analysis (7) whose valence will change in physiologic $\mathrm{pH}$. Two factors determine whether an ion will contribute to the $\mathrm{pH}$-dependent change in anion gap: $\mathrm{pK}$ and plasma concentration of the ion. A titratable substance with a pK of 7.4 will increase its anionic composition by $25 \%$ when the $\mathrm{pH}$ goes to 7.5 but if this substance exists in micromolar quantities in the plasma, it will have minimal impact on the anion gap. A substance with millimolar concentrations but with a $\mathrm{pK}$ of 3 will have no appreciable change in its anionic content with the same $\mathrm{pH}$ change from 7.4 to 7.5 . The ions that have clinical impact include phosphate and some organic anions that will increase their valence, and positively charged proteins such as some globulins that will decrease their valence as plasma $\mathrm{pH}$ rise. Both of these changes will result in an increase in the anion gap.

\section{Pyroglutamic Acidosis- An Uncommon Severe Anion Gap Metabolic Acidosis}

Although the bulk of the literature on this condition seems fairly recent, this is by no 
means a new clinical entity. It is most likely that pyroglutamic acidosis is just being recognized more frequently now than before. Pyroglutamic acid is natural product found in vegetables, fruits and grass and is synonymous with 5-oxoproline, glutimic acid, glutiminic acid, pyrolidone carboxylic acid, $\alpha$-aminoglutaric acid lactam, and perhaps a few other names unbeknownst to the authors (Fig 2). It is a strong acid and is a key intermediate metabolite in the $\gamma$-glutamyl cycle which will be discussed in more detail below. The plethora of aliases partially finds its way to the clinical literature so the identical condition is also called 5-oxoprolinuria. Since our interest and focus is on the systemic acid-base disturbance rather than the leakage of this substance in the urine, we will refer to this condition as pyroglutamic acidosis.

It is worth a brief mention how a neutral metabolic reaction can lead to metabolic acidosis as this pertains not only to pyroglutamic acid but the much more common lactic and ketoacidoses. This is illustrated in Figure 3. Metabolic cascades that are neutral may contain intermediates with low pK values so they instantly dissociate into $\mathrm{H}^{+}$and the corresponding anion (shown as $\beta$-hydroxybutyrate in Fig 3) while transiting through the pathway. The $\mathrm{H}^{+}$is buffered by $\mathrm{HCO}_{3}{ }^{-}$and indeed a momentary "acidosis" develops. However, as the base is metabolized, $\mathrm{H}^{+}$is consumed in equimolar quantity as prior $\mathrm{H}^{+}$generation and acid-base balance is restored with no net gain in acid or base as expected for a neutral metabolic reaction. However, two scenarios can convert this neutral reaction to an acid-generating process and resultant metabolic acidosis (Fig 3). First is when the generation of intermediate with the low pK far exceeds that of the consumption of this intermediate. This dysequilibrium per se can lead to accumulation of acid in the extracellular fluid. In addition, if the fate of the intermediate base (such as $\beta$-hydroxybutyrate in Fig 3) is to go to a sink rather than metabolized, the opportunity of consuming the $\mathrm{H}^{+}$and regenerating the base is foregone. An example of such a sink for the example given in Fig 3 is renal excretion of $\beta$-hydroxybutyrate as a $\mathrm{Na}^{+}$and/or $\mathrm{K}^{+}$salt. These two mechanisms can work in conjunction. This scheme can be applied to any metabolic reaction with intermediate products with low pKa's. 
To fully understand pyroglutamic acid, one needs to revisit the $\gamma$-glutamyl cycle. The $\gamma$ glutamyl cycle has been proposed to facilitate amino acid transport into cells whereas the product glutathione has a myriad of functions including the detoxification of reactive oxygen species which are particularly important in the mitochondria. Glutathione is a tripeptide (glutamic acidcysteine-glycine) found in virtually all cells except perhaps neurons (Fig 2). The characteristic peptide bond between glutamate and cysteine is on the $\gamma$ rather than the usual $\alpha$-carboxyl group that renders the bond resistant to peptidases but an excellent substrate for $\gamma$-glutamyl transpeptidase. Figure 4 summarizes the $\gamma$-glutamyl cycle. Most of the enzymes and reactions in this cycle have been confirmed by naturally occurring genetic defects and/or a wide variety of inhibitors for each enzymes $(55,56)$. Glutathione acts a donor of the $\gamma$-glutamyl group to a variety of amino acids via the action of $\gamma$-glutamyltranspeptidase on the cell membrane. The $\gamma$ glutamylaminoacid is taken up into the cell and with release of the $\gamma$-glutamyl group as a lactam ringed structure, pyroglutamic acid, which is the only strong acid in this cycle. The ring is opened to glutamic acid, which then combines with cysteine to form $\gamma$-glutamylcycteine. The enzyme is $\gamma$-glutamylcycteine which is regulated tightly by glutathione levels. $\gamma$ glutamylcycteine acquires a glycine to regenerate glutathione and completes the cycle. Both congenital enzymatic defects as well as acquired conditions can lead to pyroglutamic acidosis (see below).

Regardless of the cause, the clinical syndrome is characterized by acute onset of severe metabolic acidosis of widened anion gap. Pyroglutamic acidosis has been known as a complication of congenital glutathione or 5-oxoprolinase deficiency (57-61). Acquired forms of pyroglutamic acidosis has been in the literature for more over a decade associated with conditions such as dietary glycine deficiency from malnutrition or pregnancy, type 2 diabetes, hydrolyzed low lactose formula, and several medications such as antibiotics, vigabatrin, and acetaminophen (62-76). In the largest series by Pitt and Hauser, the bicarbonate can be as low as 
$3 \mathrm{mEq} / \mathrm{L}$ and anion gap as high as $39 \mathrm{mEq} / \mathrm{L}$ (73). The majority of cases showed a temporal relationship between the acetaminophen and/or antibiotic administration and the metabolic acidosis with subsequent resolution upon cessation of therapy.

Since this cycle is operative all the time, what is responsible for the sudden massive increase in one of its metabolic intermediate from $50-60 \mu \mathrm{M}$ to more than $30 \mathrm{mM}$ and from its baseline $20-25 \mu \mathrm{mol} / \mathrm{kg} / 30 \mathrm{mins}$ flux (77) to 10 fold that amount required to cause the clinical syndrome. Mutation in 5-oxoprolinase obviously leads to excess pyroglutamic acid. Glutathione synthetase deficiency will decrease glutathione and release the inhibition of $\gamma$-glutamylcycteine synthetase. The excess $\gamma$-glutamylcycteine will have no exit at the glutathione synthetase step but will get default to its only other metabolic pathway as a general $\gamma$-glutamylcycteine amino acid and be converted by $\gamma$-glutamylcycteine cyclotransferase to pyroglutamic acid. Glycine deficiency will likewise inhibit the glutathione synthetase step and create the same effect. Acetaminophen will deplete glutathione and exert a similar effect. It is unclear how beta-lactam antibiotics exert its effect but seeing the variety of structures of the various inhibitors of the $\gamma$ glutamyl cycle enzymes (56), it is not surprising that antibiotics may serve as pseudosubstrate competitive inhibitors. The same reasoning may apply to vigabatrin. The real incidence of increased pyroglutamic acid excretion in patients taking acetaminophen is really unknown but it is reasonable to assume that most patients either do not or only have very mild elevations of pyroglutamic acid generation. It is highly likely that there are multiple factors involved in the generation of the syndrome. There may be polymorphisms in the general population in terms of genetic susceptibility and the $\gamma$-glutamyl cycle may be impaired at multiple points by malnutrition, sepsis, antibiotics and acetaminophen administration. The syndome can be reproduced in rodents with high doses of acetaminophen and can be reversed by preventing cysteine depletion (78).

Since most if not all the patients with pyroglutamic acidosis has normal renal function, 
one would expect that the renal response to this acid load is adequate. A reasonable first approximation is to assume this condition to be an overproduction acidosis. Kamel and Halperin have pointed out that the renal response to an acute massive elevation in acid generation is quantitatively irrelevant because the rapid change does not allow adequate time for the kidney to adapt and the magnitude exceed the kidney's capacity $(79,80)$. Unfortunately, in none of the reports cited above was renal net acid excretion measured. At the moment, one makes the $a$ priori assumption that renal acidification is maximized and categorizes pyroglutamic acidosis as an overproduction acidosis but the data for this still forthcoming. One can envision that the increased production can be coupled with decreased renal net acid excretion. The pyroglutamate absorbed from the proximal tubule lumen (81-83) as well as that generated in the proximal tubule cell by the $\gamma$-glutamyl cycle (84), can both be converted to glutamate by renal 5oxoprolinase (85) and glutamate is an intermediate in the pathway for ammoniagenesis between glutamine and $\alpha$-ketoglutarate (79). An oversupply of pyroglutamate in the proximal tubule can theoretically suppress ammoniagenesis.

An interesting comparison can be made between two overproduction acidoses: Hippuric acidosis and pyroglutamic acidosis. The hippuric acidosis from toluene intoxication (gluesniffing) appears to generate a near but submaximal renal acidification response qualifying it as an overproduction acidosis (86). The characteristic of hippuric acidosis is that the plasma anion gap typically remains normal (86) or only very slightly elevated. In contrast, pyroglutamate appears to accumulate in the plasma hence disturbing the anion gap.

While hippurate is processed by a filtration-secretion made in the proximal tubule (87, 88), the renal handling of pyroglutamate is not as clear but the following analysis supports a filtration-reabsorption system (Fig 5). Pyroglutamic acid is taken up from proximal tubule brush border membrane vesicles via a $\mathrm{Na}^{+}$-dependent carrier (81-83). Interestingly, pyroglutamic acid seems to share transporter mechanisms with the neutral amino acid $\mathrm{B}^{0+}$ rather than anionic amino 
acids $\mathrm{X}^{-\mathrm{A}, \mathrm{G}}$ system (83). The interaction between pyroglutamic acid and neutral amino acid may be more complex than sharing a transporter as pyroglutamic acid appears to stimulate rather than compete with the $\mathrm{B}^{0+}$ neutral amino acid transport in the blood brain barrier (89). Since there is no basolateral uptake of pyroglutamic acid from the plasma to the renal tubule via the basolateral membrane, the apical $\mathrm{Na}^{+}$-coupled uptake likely results in reabsorption of filtered pyroglutamate although the relatively low affinity of $20 \mathrm{mM}(83)$ is unlikely to permit complete uptake.

\section{Conclusion}

In this short review, the authors wish to convey and emphasize the concept that the clinical anion gap is not a mere reflection of leftover anions of strong acids that has decomposed bicarbonate. The utility of the anion gap should exceed way beyond that of the differential diagnosis of metabolic acidosis. Once again, emphasis is placed on the fact that the clinical anion gap is the difference between the unmeasured anions and the unmeasured cations and any numerical analysis of this entity needs to encompass this equation into consideration. Only by exploring all the possible factors that can influence unmeasured cations and unmeasured anions can one truly extract useful information from the clinical anion gap. Previously unrecognized or poorly described disorders of the anion gap such as pyroglutamic acidosis will continue to emerge in clinical medicine. 


\section{Acknowledgment}

The authors' research programs are supported by the National Institutes of Health (R01-DK-

48482, R01-DK-54392 and PO1-DK20543 to OWM) and the Department of Veteran Affairs Research Service (OWM). DF was the recipient of a fellowship from the Swiss National Science Foundation. 


\section{Figure Legends}

Figure 1: Hyponatremia from non-aqueous plasma volume. The use of ion-selective electrodes that measures $\mathrm{Na}^{+}$activity theoretically rids the entity of pseudohyponatremia from non-aqueous volumes in the plasma if the plasma is never diluted. However, automated aspirator/dilutors perpetuates this problem. The top panel depicts the situation in normal plasma. A 10x dilution of plasma with water dilutes $\left[\mathrm{Na}^{+}\right]$to $14 \mathrm{mEq} / \mathrm{L}$ and the back-calculation with a factor of 10 recapitulates the original plasma $\left[\mathrm{Na}^{+}\right]$of $140 \mathrm{mEq} / \mathrm{L}$. The bottom panel depicts plasma with normal $\left[\mathrm{Na}^{+}\right]$of 140 but an excessive non-aqueous space. The aspirator will take an erroneously smaller amount of aqueous plasma and dilute it $10 \mathrm{x}$ with water yielding a final $\left[\mathrm{Na}^{+}\right]$of 11.2 $\mathrm{mEq} / \mathrm{L}$. Back calculation with the same dilution factor gives a plasma $\left[\mathrm{Na}^{+}\right]$of $112 \mathrm{mEq} / \mathrm{L}$.

Figure 2: Structures of pyroglutamic acid and glutathione. Pyroglutamic acid shares the same formula $\left(\mathrm{C}_{5} \mathrm{H}_{7} \mathrm{NO}_{3}\right)$ as glutamic acid but it contains a lactam ring with a highly dissociable carboxyl group. Unlike proline which exist as a zwitterion at $\mathrm{pH} 7.4$, pyroglutamic acid is completely dissociated at $\mathrm{pH}$ 7.4. Glutathione is a tripeptide of glutamic acid, cysteine and glycine but the peptide bond between glutamate and cysteine is on the $\gamma$ - instead of $\alpha$-carboxy group of glutamate $(*)$ hence the name $\gamma$-glutamylcysteinylglycine..

Figure 3: Metabolic acidosis from a neutral metabolic reaction. A metabolic reaction with neutral (acid-base viewpoint) starting substrates and end products such as the breakdown of triacylglycerol into carbon dioxide. One intermediate product is a strong acid such as $\beta$ hydroxybutyric $(\mathrm{H} \beta \mathrm{HB})$ acid which dissociates instantaneously in the plasma consuming $\mathrm{HCO}_{3}{ }^{-}$ which will be regenerated when the $\beta$-hydroxybutyrate anion $\left(\beta \mathrm{HB}^{-}\right)$is metabolized. The bottom panel shows the two ways by which metabolic acidosis can be generated. 1 . The rate of the acid generation step exceeds the acid-consumin step. 2. The base is lost from the body before it is metabolized. 
Figure 4: Gamma-glutamyl cycle and pyroglutamic acidosis. Enzymes of the $\gamma$-glutamyl cycle is shown in italics. Monogenic defects have been described in four of the enzymes (asterisk). A variety of amino acids can acquire a $\gamma$-glutamyl group donated by glutathione via $\gamma$ glutamyltransferase. Upon release of the amino acid, the $\gamma$-glutamyl group is released as a lactam structure which is pyroglutamic acid (or oxoproline). Pyroglutamic acid is a strong monocarbolylic acid and is completely dissociated at plasma $\mathrm{pH}$. The ring structure is opened to release glutamate by 5-oxoprolinase. Glutathione is regenerated by subsequent addition of cysteine and glycine. Since $\gamma$-glutamylcycteine is a true $\gamma$-glutamyl amino acid, it is a substrate for $\gamma$-glutamyl cyclotransferase (\#) which is a high capacity enzyme. Glutathione shuts off its own synthesis not at the immediate proximal step but via negative feedback to inhibit $\gamma$ glutamylcycteine synthetase.

Figure 5: A consideration of two conditions of acid overproduction and a theoretical explanation of the changes in the plasma anion gap. Two metabolic acidoses and the renal handling of their anions- hippurate $\left(\mathrm{Hp}^{-}\right)$and pyroglutamate $\left(\mathrm{pG}^{-}\right)$. Two assumptions are made. Both hippuric acid and pyroglutamic acid consumes a similar amount of $\mathrm{HCO}_{3}{ }^{-}$when added to plasma. Renal ammoniagenesis is stimulated but inadequate to regenerate the consumed $\mathrm{HCO}_{3}{ }^{-}$ hence the acidosis. However the excretion of $\mathrm{Hp}^{-}$is much more efficient than $\mathrm{pG}^{-}$resulting in normalization of the anion gap in hippuric and not pyroglutamic acidosis. 
Table 1: Plasma values of Chrysemys picta marginate (diving turtle)

\section{Before diving}

\section{Anoxic water $x 150$ days}

\section{Concentration in $\mathrm{mM}$}

$\begin{array}{llll}\text { Sodium } & 117 & 99 & \\ \text { Chloride } & 73 & 44 & \\ \text { Bicarbonate } & 39 & 5 & \Delta=34 \\ \text { Anion Gap } & 5 & 50 & \Delta=45\end{array}$

Unmeasured anions

$\begin{array}{lll}\text { Lactate } & 4 & 185 \Delta=181\end{array}$

Unmeasured cations

Potassium

2

10

Total Magnesium*

2.6

12

Total Calcium*

3.7

59

*Not all in ionized form. Some exist in charged or uncharged soluble complexes so a valence of 2 should not be used for conversion from mmole to mEq. Values taken from Reese et al. Resp Physiol 124:43-50, 2000. 
Table 2: Changes in anion gap in bromide intoxication

Sensitivity of assay to halides

Anion gap in bromide intoxication

$\begin{array}{ll}\mathrm{Cl}^{-}=\mathrm{Br}^{-} & \text {No change } \\ \mathrm{Br}^{-}>\mathrm{Cl}^{-} & \text {Low anion gap } \\ \mathrm{Cl}^{-}>>\mathrm{Br}^{-} & \text {High anion gap }\end{array}$




\section{References}

1. Emmett ME \& Narins RG, Clinical Use of the Anion Gap. Medicine 1977;56:38-54.

2. Oh MS \& Carroll HJ. Current concepts: The anion gap. New England Journal of Medicine 1977;297:814-817.

3. Winter SD, Pearson J \& Gabow PA: The fall of the serum anion gap in the diagnosis of acid base disorders. Archives of Internal Medicine 1990; 150:311-313.

4. Reese SA, Crocker CE, Jackson DC et al. The physiology of hibernation among painted turtles. Respiration Physiology 2000;124:43-50.

5. Jackson DC. Living without oxygen: lessons from the freshwater turtle. Comparative Biochemistry and Physiology 2000;125:299-315.

6. Jackson DC, Crocker, \& Ultsch GR. Bone and shell contribution to lactic acid buffering of submerged turtles Chrysemys picta belli at $3^{\circ} \mathrm{C}$. American Journal of Physiology 2000;278:R1564-R1571.

7. Stewart PA. Modern quantitative acid-base chemistry. Canadian Journal of Physiology and Pharmacology 1983;61:1444-1461.

8. Constable PD. A simplified strong ion model for acid-base equilibria: application to horse plasma. Journal Applied Physiology 1997;83:297-311.

9. Constable PD. Clinical assessment of acid-base status: comparison of the HendersonHasselbalch and strong ion approaches. Veterinary Clinical Pathology 2000;29:115-128.

10. Boyle M, \& Baldwin I. Introduction to an alternate view of acid/base balance: the strong ion difference or Stewart approach. Australian Critical Care 2002;15:14-20.

11. Gabow PA. Disorders associated with an altered anion gap. Kidney International 1985; 27:472-483.

12. Weisberg LS. Pseudohyponatermia: a reappraisal. American Journal Medicine 1989; 86:315-318.

13. Faye S \& Payne RB. Rapid measurement of serum water to assess pseudohyponatremia. Clinical Chemistry 1986;32:983-986.

14. Jurado RL del Rio C, Nassar G et al. Low anion gap. Southern Medical Journal 1998;91:624-629.

15. McDonald DA. Effect of protein and triglycerides on serum sodium and potassium values 
obtained by the Kodak dry film potentiometric technique. Canadian Journal Medical Technology 1986:48:146

16. Keshegina AA. Decreased anion gap in diffuse polyclonal hypergammaglobulinemia. New England Journal Medicine 1978: 299:99-100.

17. Vader HL \& Vink CLJ. The influence of viscosity on dilution methods: Its problems in the determination of serum sodium. Clinica. Chimica. Acta 1975; 65:379-388.

18. Gamble JL. Chemical Anatomy, Physiology and Pathology of Extracellular Fluid: A Lecture Syllabus. $6^{\text {th }}$ Edition, Harvard University Press, Cambridge, Mass., 1960, p.131

19. Figge J, Jabor A, Kazda A, \& Fencl V. Anion gap in hypoalbuminemia Critical Care Medicine 1998;26:1807-1810.

20. Durward A, Mayer A, Skellet S et al. Hypoalbuminaemia in critically ill children. Archives of the Diseases of the Child 2003;88:419-422.

21. Walser M \& Rahill WJ. Renal tubular reabsorption of bromide compared with chloride. Clinical Science 1966;30:191-205.

22. Rothenberg DM, Berns AS, Barkin R et al. Bromide intoxication secondary to pyridostigmin bromide therapy. Journal of American Medical Association 1990;263:1121-1122.

23. Blume RS MacLowry JD \& Wolff SM. Limitations of the chloride determination in the diagnosis of bromism. New England Journal of Medicine1968; 279:593-595.

24. Driscoll JL \& Martin HF. Detection of bromism by an automated chloride methods. Clinical Chemistry 1966;12:314-318.

25. Arai K, Kusu F, Noguchi N et al. Selective determination of chloride and bromide ions in serum by cyclic voltammetry. Analytical Biochemistry 1996;240:109-113.

26. Saller B, Fink H, \& Mann K. Kinetics of acute and chronic iodine excess. Experimental and Clinical Endocrinology and Diabetes. 1998;106(Suppl 3):S34-S38.

27. Murray T, Long W, \& Narins RG. Multiple Myeloma and the Anion Gap. New England Journal Medicine 1975;292:574-575.

28. Paladini G \& Sala PG. Effect of chemotherapy on the anion gap in multiple myeloma. Acta Haematologica 1981;66:31-34.

29. Frohlich J, Adam W, Golbey MJ \& Bernstein M. Decreased anion gap associated with monoclonal and pseudomonoclonal gammopathy. Canadian Medical Association Journal 1976;114:231-232. 
30. Witte DL, Rodgers JL \& Barrett DA 2nd. The anion gap: its use in quality control. Clinical Chemistry 1976;22:643-646.

31. Keshgegian AA. Anion gap and immunoglobulin concentration. American Journal Clinical Pathology 1980;74:282-284.

32. De Troyer A, Stolarczyk A, De Beyl DZ, et al. Value of anion-gap determination in multiple myeloma New England Journal of Medicine 1977;296:858-860.

33. Paladini G \& Sala PG. Anion gap in multiple myeloma Acta Haematologica. 1979;62:148-152.

34. O'Connor DT \& Stone RA. Hyperchloremia and negative anion gap associated with polymyxin B administration. Archives of Internal Medicine 1978; 138:478-480

35. Jacobson HR. Effects of $\mathrm{CO}_{2}$ and acetazolamide on bicarbonate and fluid transport in rabbit proximal tubules. American Journal of Physiology 1981;240:F54-F62.

36. Cogan M. Effects of acute alterations in $\mathrm{PCO}_{2}$ on proximal $\mathrm{HCO}_{3}, \mathrm{Cl}-$, and $\mathrm{H}_{2} \mathrm{O}$ reabsorption. American Journal of Physiology 1984;246:F21-F26.

37. Jacobson HR. Medullary collecting duct acidification: effects of potassium, $\mathrm{HCO}_{3}$ concentration, and $\mathrm{PCO}_{2}$. Journal of Clinical Investigation 1984;74:2107-2114.

38. Bengele HH, McNamara ER, Schwartz JH, et al. Acidification adaptation along the inner medullar collecting duct. American Journal of Physiology 55:F1155-F1159.

39. Gennari FJ, Goldstein MB, \& Schwartz WB. The nature of the renal adaptation to chronic hypocapnia. Journal Clinical Investigation 1972;51:1722-1730.

40. Dempsey JA, Forster HV, \& DoPico GA. Ventilatory acclimatization to moderate hypoxemia in man. Journal Clinical Investigation 1974;53:1091-1100.

41. Forster HV, Dempsey JA, \& Chosy LW. Incomplete compensation of CSF $\left[\mathrm{H}^{+}\right]$in man during acclimatization to high altitude (4300 m). Journal Applied Physiology 1975;38:10671072 .

42. Gledhill N, Beirne GJ, \& Dempsey JA. Renal response to short-term hypocapnia in man. Kidney International 1975;8:376-386.

43. Giebisch G, Berger L, \& Pitts RF. The extrarenal response to acute acid-base disturbances of respiratory origin. Journal Clinical Investigation 1955;34:231-245.

44. Eichenholz A, Mulhausen RO, Anderson WE, et al. Primary hypocapnia: a cause of metabolic acidosis. Journal of Applied Physiology 1962;17:283-288.

45. Plum F, Posner JB. Blood and cerebrospinal fluid lactate during hyperventilation. American 
Journal of Physiology 1967;212:864-870.

46. Zborowska-Sluis DT, Dossetor JB. Hyperlactatemia of hyperventilation. Journal of Applied Physiology 1967;22:746-755.

47. Engle K, Kildeberg P, Winters RW. Quantitative displacement of acid-base status in acute hypocapnia. Scandinavian Journal of Clinical and Laboraotry Investigation 1969;23:5-17.

48. Eldridge F, Salzer J. Effect of respiratory alkalosis on blood lactate and pyruvate in humans. Journal of Applied Physiology 1967;22:461-468.

49. Arbus GS, Herbert LA, Levesque PR, et al. Characterization and clinical application of the "significance band" for acute respiratory alkalosis. New England Journal of Medicine 1969;280:117-123.

50. McAuliffe JJ, Lind LJ, Leith DE, Fencl V. Hypoproteinemic alkalosis. American Journal of Medicine 1986;81:86-90.

51. Figge J, Rossing TH \& Fencl V. The role of serum protein in acid-base equilibria. Journal of Laboratory and Clinical Medicine 1991;117:453-467.

52. Gianazza E, Miller I, Eberini I, \& Castiglioni S. Low-tech electrophoresis, small but beuatiful, abd effective: E; ectrophoretic titration curves for proteins. Electrophoresis 1999;20:1325-1328.

53. Paulson Effect of acute $\mathrm{pH}$ change on serum anion gap. Journal of American Society of Nephrology 1996;7:357-363.

54. Spielberg SP, Garrick MD, Corash LM, et al. Biochemical heterogeneity in glutathione synthetase deficiency. Journal Clinical Investigation 1978;61:1417-1120.

55. Ristoff E, Larsson A. Patients with genetic defects in the gamma-glutamyl cycle. Chemical Biologic Interaction. 1998;111:114-121.

56. Meister A, Griffith OW, Novogrodsky A, \& Tate SS. New aspects of glutathione metabolism and translocation in mammals. Ciba Foundation Symposium 1979;72:135-1361

57. Spielberg SP, Kramer LI, Goodman SI, et al. 5-oxoprolinuria: biochemical observations and case report. Journal of Pediatrics 1977;91:237-241.

58. Spielberg SP, Garrick MD, Corash LM, et al. Biochemical heterogeneity in glutathione synthetase deficiency. Journal of Clinical Investigation 1978;61:1417-1420.

59. Griffith OW, Meister A Glutathione: interorgan translocation, turnover, and metabolism. Proceeding of the National Acadamy of Science USA. 1979;76:5606-5510.

60. Larsson A, Mattsson B, Wauters EA, van Gool JD, Duran M, Wadman SK. 5-oxoprolinuria 
due to hereditary 5-oxoprolinase deficiency in two brothers--a new inborn error of the gamma-glutamyl cycle. Acta Paediatrica Scandinavia. 1981;70:301-308.

61. Al-Jishi E., Meyer BF, Rashed MA et al. Clinical, biochemical, and molecular characterization of patients with glutathione synthetase deficiency. Clinical Genetics 1999;55:444-449.

62. Oberholzer VG, Wood CB, Palmer T, \& Harrison BM. Increased pyroglutamic acid levels in patients on artificial diets. Clinica Chimica Acta. 1975;62:299-304.

63. Jackson AA, Badaloo AV, Forrester T, Hibbert JM, Persaud C. Urinary excretion of 5-oxoproline (pyroglutamic aciduria) as an index of glycine insufficiency in normal man. British Journal of Nutrition 1987;58:207-214.

64. Persaud C, McDermott J, De Benoist B, Jackson AA. The excretion of 5-oxoproline in urine, as an index of glycine status, during normal pregnancy. British Journal of Obstetrics and Gynaecology. 1989;96:440-444.

65. Creer MH, Lau BW, Jones JD, Chan KM. Pyroglutamic acidemia in an adult patient. Clinical Chemistry 1989;35:684-686.

66. Meister A.Vigabatrin and urinary 5-oxoproline Lancet. 1989;2:1216.

67. Bonham JR, Rattenbury JM, Meeks A, \& Pollitt RJ. Pyroglutamicaciduria from vigabatrin. Lancet 1989;1:1452-1453.

68. Forrester TE, Badaloo V, Bennett FI, \& Jackson AA. Excessive excretion of 5-oxoproline and decreased levels of blood glutathione in type II diabetes. European Journal of Clinical Nutrition. 1990;44:847-850.

70. Pitt J. Association between paracetamol and pyroglutamic aciduria. Clinical Chemistry 1990;36:173-174.1990

71. Persaud C, Forrester T, \& Jackson AA.Urinary excretion of 5-L-oxoproline (pyroglutamic acid) is increased during recovery from severe childhood malnutrition and responds to supplemental glycine. Journal of Nutrition. 1996;126:2823-2830.

72. Jackson AA, Persaud C, Hall M, et al. Urinary excretion of 5-L-oxoproline (pyroglutamic acid) during early life in term and preterm infants. Archives of Diseases of the Child Fetal and Neonatalogy 1997;76:F152-F157.

73. Pitt JJ, Hauser S. Transient 5-oxoprolinuria and high anion gap metabolic acidosis: clinical and biochemical findings in eleven subjects. Clin Chem. 1998 Jul;44(7):1497-503.

74. Croal BL, Glen AC, Kelly CJ, Logan RW. Transient 5-oxoprolinuria (pyroglutamic aciduria) with systemic acidosis in an adult receiving antibiotic therapy. Clinical Chemistry

1998;44:336-340 
75. Mayatepek E.5-Oxoprolinuria in patients with and without defects in the gamma-glutamyl cycle. European Journal of Pediatrics 1999;158:221-225.

76. Dempsey GA, Lyall HJ, Corke CF, Scheinkestel CD. Pyroglutamic acidemia: a cause of high anion gap metabolic acidosis Critical Care Medicine. 2000;28:1803-1807.

77. Metges CC, Yu YM, Cai W et al. Plasma L-5-oxoproline carbon and nitrogen kinetics in healthy young adults. Journal of Nutrition 1999;129:1998-2004.

78. Ghauri FY, McLean AE, Beales D, et al. Induction of 5-oxoprolinuria in the rat following chronic feeding with $\mathrm{N}$-acetyl 4-aminophenol (paracetamol). Biochemical Pharmacology 1993 ;46:953-957.

79. Halperin ML, Kamel KS, Ethier JH et al. Biochemistry and physiology of ammonium excretion. In: The Kidney: Physiology and Pathophysiology. Editors: Seldin DW, Giebisch. $2^{\text {nd }}$ Edition, Raven Press New York NY, 1992; pp. 2645-2680

80. Kamel KS, Briceno LF, Sanchez MI, et al. A new classification for renal defects in net acid excretion American Journal of Kidney Disease 1997;29:136-146.

81. Ganapathy V \& Leibach FH. Electrogenic transport of 5-oxoproline in rabbit renal brush-border membrane vesicles: Effect of intravesicular potassium. Biochimica Biophysica Acta 1983; 732:32-40.

82. Ganapathy V, Roesel RA, Howard JC, \& Leibach FH. Interaction of proline, 5-oxoproline, and pipecolic acid for renal transport in the rabbit. Journal of Biological Chemistry 1983;

258:2266-2272.

83. Ganapathy V, Roesel RA, \& Leibach FH. Transport of 5-oxoproline into rabbit renal brush border membrane vesicles. Biochemical Biophysical Research Communication 1982;105:28-35.

84. Bridges RJ \& Meister A. gamma-Glutamyl amino acids. Transport and conversion to 5-oxoproline in the kidney. Journal of Biological Chemistry 1985;260:7304-7308.

85. Meister A \& Griffith OW, Williamson JM. 5-Oxo-L-prolinase from rat kidney. Methods in Enzymology 1985;113:445-451.

86. Carlisle EJ, Donnelly SM, Vasuvattakul S, et al. Glue-sniffing and distal renal tubular acidosis: sticking to the facts. Journal of American Society of Nephrology 1991;1:1019-1027.

87. Ullrich KJ. Renal transporters for organic anions and organic cations. Structural requirements for substrates. Journal of Membrane Biology 1997;158:95-107.

88. Burckhardt G, Bahn A, \& Wolff NA. Molecular physiology of renal p-aminohippurate secretion. News in Physiologic Science. 2001;16:114-118. 
89. Lee WJ, Hawkins RA, Peterson DR, \& Vina JR. Role of oxoproline in the regulation of neutral amino acid transport across the blood-brain barrier. Journal of Biological Chemistry 1996;271:19129-19133. 


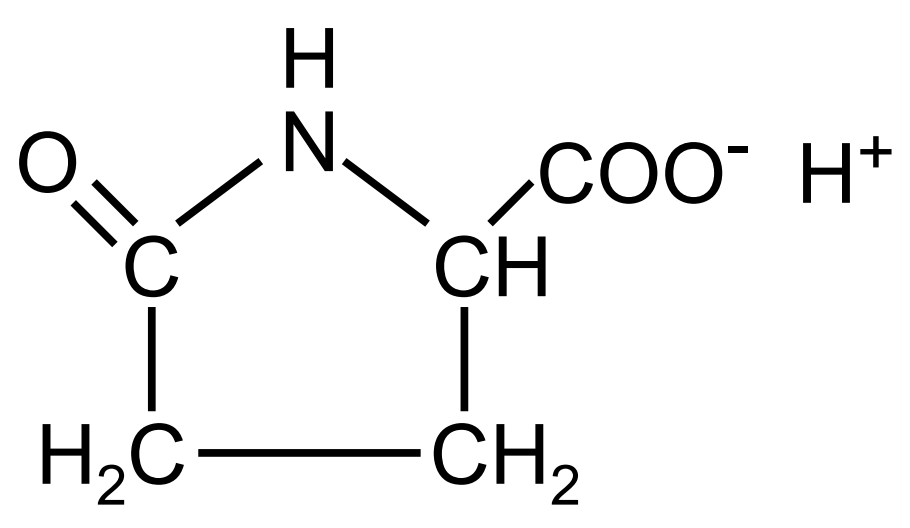

Pyroglutamic acid

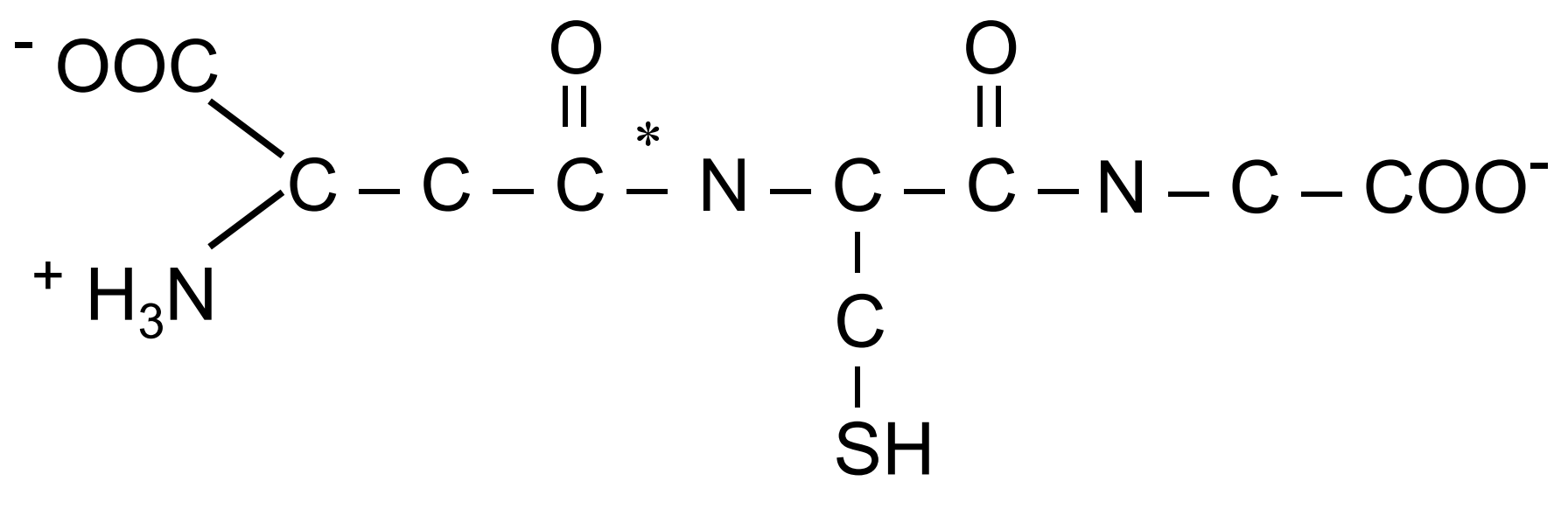
$\checkmark$

Glutathione ( $\gamma$-glutamylcysteinylglycine) 

(acid/base pair)

Triacylglycerol

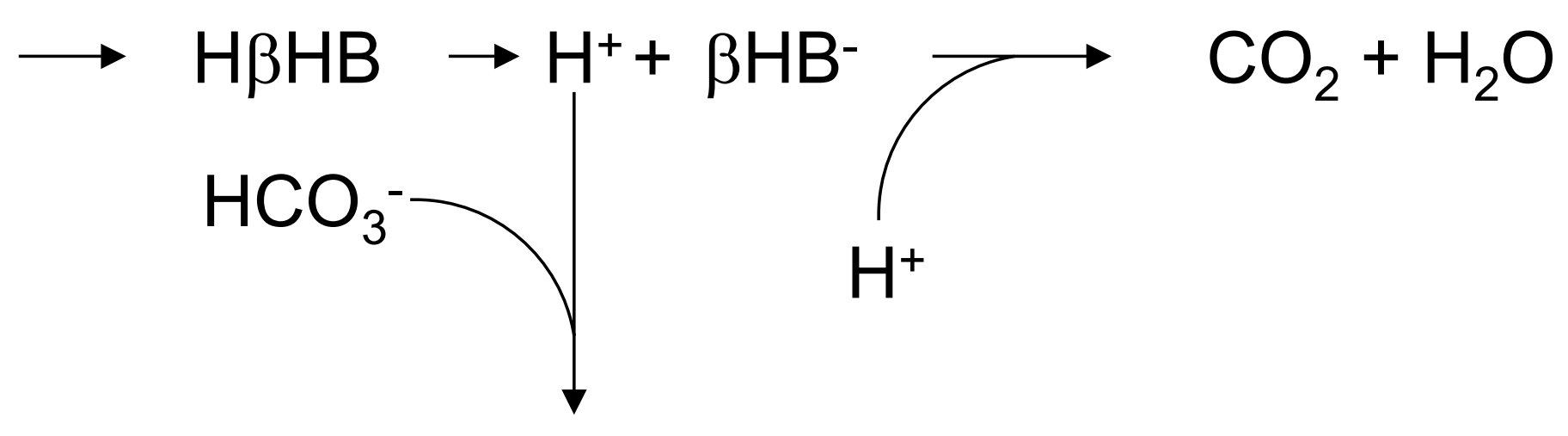

1. Dysequilibrium

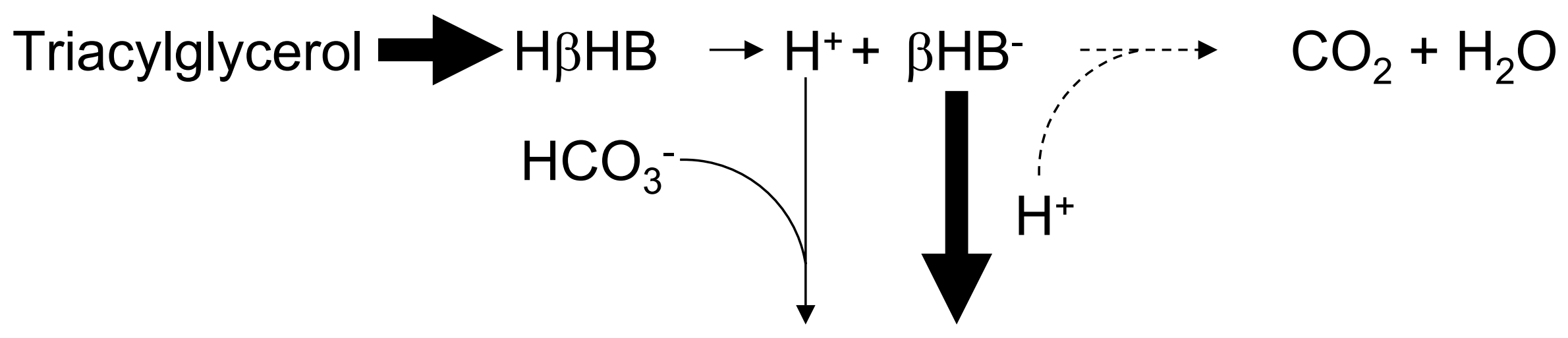

2. Sink for base 


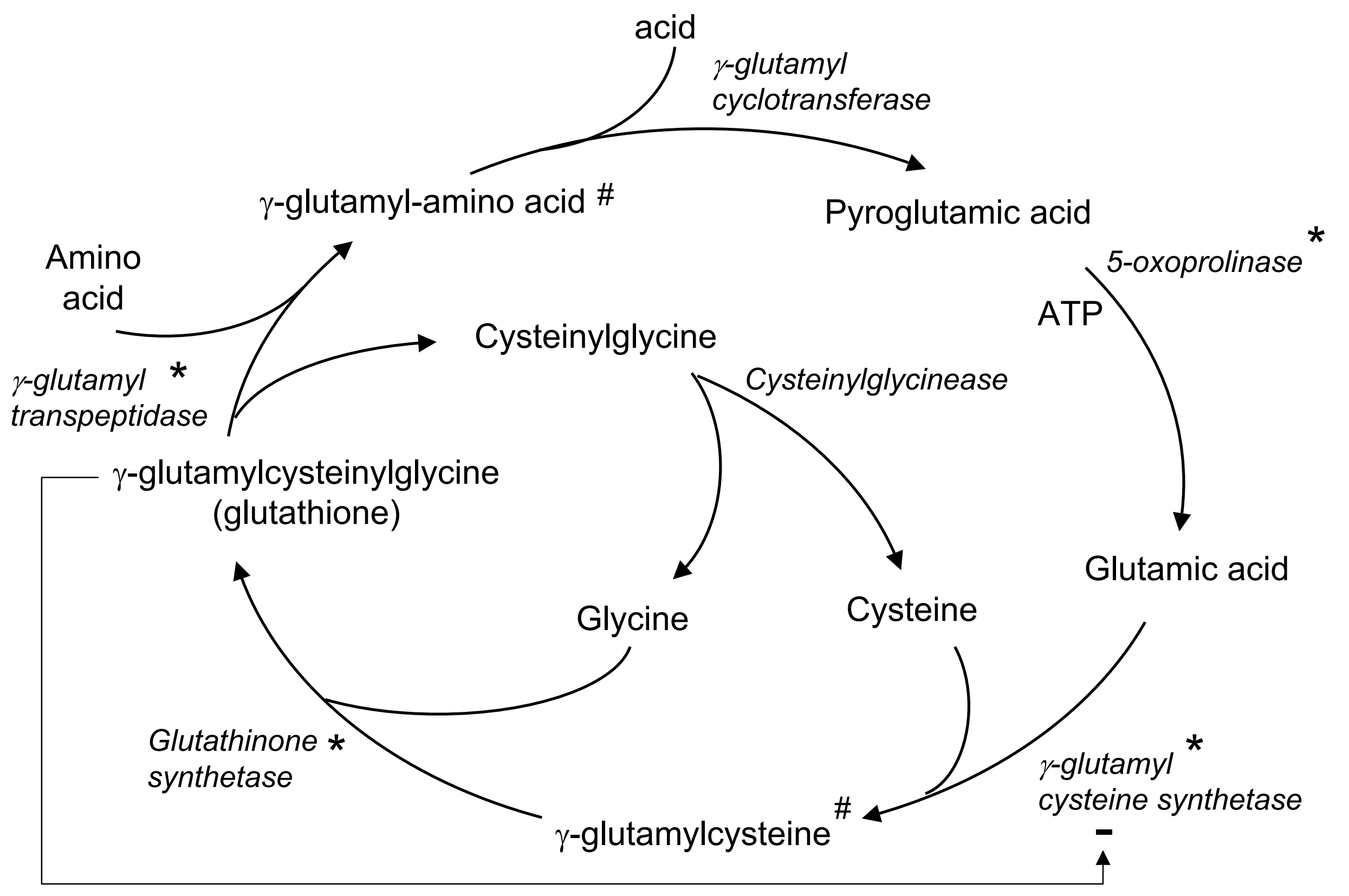


